\title{
Predictive factors of survival rate in oral squamous cell carcinoma: a retrospective study in Iran
}

\author{
Narges Gholizadeh, ${ }^{a}$ Hanieh Sadat Emami Razavi, ${ }^{b}$ Gholamali Jafari Haftkhani, ${ }^{c}$ and Nafiseh Sheykhbahaei ${ }^{a}$
}

\author{
aral \& Maxillofacial Medicine, School of Dentistry, Tehran University of Medical Science, Tehran, Iran. \\ bDepartment of Operative Dentistry, School of Dentistry, Alborz University of Medical Science, Karaj, Iran. \\ cDepartment of Ear, Nose, Throat and Head and Neck Surgery, Baqiyatallah University of Medical Science, Tehran, Iran. \\ Correspondence to Nafiseh Sheykhbahaei (email: dsheykhbahaei@gmail.com). \\ (Submitted: 12 January 2019 - Revised version received: 27 February 2019 - Accepted: 05 March 2019 - Published online: 26 April 2019)
}

\begin{abstract}
Objective Oral cancer as a part of this collection plays an important role in the burden of diseases, especially in South Asia. Despite numerous advances in the treatment of cancers, oral cancer is one of the top 10 causes of death due to delayed detection. Given the role of the environmental and climatic conditions in cancers, there is a need for epidemiological studies in different geographic areas.

Methods In this study, data were collected through medical records of the patients with oral cancer during 2009-2012 in the archives of the Cancer Department of the Ministry of Health of the Islamic Republic of Iran. The follow-up of 82 cases was possible in the study. Data collection were performed from questionnaire. The data were analyzed by SPSS version 20. The Kaplan-Meier survival curves were utilized and, moreover, the corresponding influential factors were examined using the Cox regression test.

Results The overall 5-year survival rate was $40.24 \%$ (SE $=5.5$ ). The most widely used treatment method was a combination of three methods of surgery, radiation therapy, and chemotherapy. The gender of $64.63 \%$ of patients were male. The mean age of patients were 66.1 years. The age and regular periodic follow-up had a statistically significant relation with the survival rate $(P=0.02,0.03)$.

Conclusion Oral cancers require special attention due to high prevalence and overall survival rates. Training oral health care providers, and periodic follow-up can be an important step in increasing the survival of these patients.

Keywords oral squamous cell carcinoma, delay, survival rate
\end{abstract}

\section{Introduction}

Nowadays, increasing the world's population and the average age, promoting life expectancy, improving the health status and high exposure to carcinogenic risk factors have led to an increase in the global incidence of cancer. Oral cancer as a part of this collection plays an important role in the burden of diseases, especially in South Asia. ${ }^{1,2}$ The World Health Organization (WHO) in 2012 has emphasized the prevention of cancer and the promotion of quality-of-life of cancer patients. Nearly $40 \%$ of deaths from cancer will be prevented by controlling risk factors, and whose one-third burden will be reduced by timely diagnosis and treatment. ${ }^{3}$ Cancer is the third most common cause of death in Iran with more than 30,000 deaths annually. ${ }^{4}$ About $5 \%$ of all cancers occur in the head and neck and about half of these are in the oral cavity. Squamous cell carcinoma (SCC) is the most common head and neck cancer whose incidence has been reported to have descending trend over the last three decades in the world. ${ }^{5}$ According to global data on the incidence of oral cancer map in 1997, Iran, along with other South Asian countries, is considered as one of the countries with a high rate of oral cancer with an outbreak of between 20 and 36.3 per 100,000 people. ${ }^{4}$ According to data released by the Iranian Ministry of Health in 2003, the incidence of oral cancer is among the 10 most common cancers in men and women. The oral cancers are related to tobacco use, smoking, alcohol consumption, low socioeconomic status, and poor health, and improper diet, viral infections such as HPV, poorly fitted dentures, and chronic trauma. ${ }^{6}$

The biological behavior of SCC suggests highly invasive and poor prognostic nature.

In the industrialized countries, men are two to three times more likely to be involved than women, mainly because of further exposure to the risk factors. ${ }^{1}$ The mean age of the diagnosis is 57.1 years in males and 52.5 years in females, with the highest incidence in the sixth decade of life. ${ }^{6}$

Genetic factors are now widely recognized as an etiology because the SCC is developed in children and adolescents without any risk factor.

The main anatomical areas include the tongue, the floor of mouth, the gums, the palate, and other parts of the mouth; the involvement of various oral areas is affected by geographical factors. For example, the most common involved area is the tongue between the European and American populations, and buccal mucus cancers among the Asian population due to a habit of chewing tobacco such as betel quid. ${ }^{7}$

Despite numerous advances in the treatment of cancers, oral cancer is one of the top 10 causes of death due to delayed detection with several causes, such as early-stage asymptomatic nature, clinical presentation similar to other lesions, and variation in clinical manifestations. Given the role of the environmental and climatic conditions in cancers, there is a need for epidemiological studies in different geographic areas.

\section{Materials and Methods}

This study was conducted in retrospective and cross-sectional design, whose data were collected through the 436 medical records of the patients with oral cancer during 2009-2012 in the archives of the Cancer Department of the Ministry of Health of the Islamic Republic of Iran. Totally, the enrolled medical records in our study were 82 out of 436 cases with a histopathologic diagnosis of oral squamous cell carcinoma, and a contact information or mailing address. All new cases of cancer detection in Iran refer to cancer registry centers from pathology labs through the forms prepared by the Ministry of 
Health since 2003, consisting of demographic profiles, diagnostic times and pathological parameters of the disease. Other items included in this form are the location of the lesion, the degree of differentiation and degree of invasion with the special codes. Information of our patients was gathered by a checklist designed as two groups of questions that were completed by the information in Cancer Registration Center forms and telephone by patients or relatives, including survival status, treatment method and delayed time. In collecting patient information, the lack of a response to three times of phone call with a 2-week interval resulted in the use of postal services, and in the absence of a repeated response, the use of the medical record available at the Cancer Institute of Imam Khomeini Hospital. Meanwhile, if any, the relatives were asked to explain about the causes of death in the deceased subjects, which classified into two groups of cancer complications and other causes such as accidents or heart attacks. Concerning a delay studied within the two groups of $<4$ weeks and $>4$ weeks, the patient delay was defined as interval from the onset of disease symptoms to the first patient referral, and professional delay as interval from the first patient referral to diagnosis.

This study was approved by The Ethics Committee of Tehran University of Medical Science. The SPSS version 20 software was used to analyze the attained data using descriptive statistics of tables and figures, and the Kaplan-Meier curve to evaluate the survival, as well as the simple and multivariate Cox regression test to assess the effect of the studied factors on survival rate. In all tests, $P<0.05$ was considered as statistically significant difference.

\section{Results}

Our subjects included 53 (64.63\%) males and 29 (35.37\%) females and $97.33 \%$ of them were married. The results showed that the prevalence of oral cancer in females was twice lower than in males.

According to Fig. $1,32.9 \%$ of patients were $\leq 60$ years and $67.1 \%$ were $>60$ years with the mean age of 66.1 years for all patients ranging from 31 to 89 years. In fact, most of our patients were living in their $6^{\text {th }}$ to $8^{\text {th }}$ decades of life. On the basis of histological grade of the lesions, $67.39 \%$ of the patients suffered from completely differentiated lesions, $17.39 \%$ had relatively differentiated lesions and $15.22 \%$ of them showed less differentiated lesions.

In this study, there were several diagnostic procedures, the participants found the risk of cancer symptoms by themselves ( $n=42,71.18 \%)$, a physician due to referral for other diseases $(n=13,22.03 \%)$ and a dentist $(n=4,6.79 \%)$. The statistical findings of the first referral in this study can be categorized according to whom the patient has referred to for the first time. The first referral of the patients was to a specialist physician $(n=41,56.18 \%)$, a general practitioner $(n=24$, $32.87 \%)$ and a dentist $(n=8,10.95 \%)$.

About delay factors in cancer management, the mean patient delay was reported to be $16.3 \pm 17.7$ weeks (approximately 4 months) ranging from a week to a year (52 weeks). A delay $<4$ weeks was $37.8 \%$ and a delay $>4$ weeks was $46.3 \%$. The mean professional delay was $4.75 \pm 5.29$ weeks ranging from a week to 28 weeks.

The patients with the oral cancers were treated in three methods, surgery, radiotherapy and chemotherapy. In many cases, the treatment of patients was a combination of two or three methods. Accordingly, the most commonly used treatment $(36.6 \%)$ for the patients was the combination of all three treatments. Surgery $15.9 \%$, chemotherapy $3.7 \%$, radiotherapy $6.1 \%$, chemoradiotherapy $11 \%$, chemosurgery $15.9 \%$, radiosurgery $7.3 \%$ and $2.4 \%$ of patients with no treatment.

The periodic follow-up was fulfilled in the hospital $(n=30,66.66 \%)$ and in the private medical clinic $(n=15$, $33.34 \%)$ and no follow-up programs $(n=0)$. An oral hygienist performed the periodic follow-up for $12.2 \%$ of the patients.

In this study, the locations of tumor were respectively the lateral borders of tongue (20.4\%), the floor of mouth (2.1\%), the base of the tongue (1.7\%), lips (1.3\%), gums and palate $(0.95 \%)$ and other parts of the mouth $(7.7 \%)$.

In this study, 49 (59.75\%) survived and 33 (40.25\%) died during the study due to the cancer complications ( $n=43$, 93.47\%) and other causes $(n=3,6.93 \%)$. As can be seen in Table 1, the 1-year overall survival rate was $91.46 \%(\mathrm{SE}=3.09)$, the 3-year overall survival rate was $53.7 \%(\mathrm{SE}=5.5)$ and the 5 -year overall survival rate was $40.24 \%(\mathrm{SE}=5.5)$.

The survival rate in females was more than in males, but the gender showed no statistically significant relationship with survival rate $(P=0.41)$. However, the age of the patients had statistically significant relationship with survival rate, meaning the reduction in the survival rate with age $(P>0.001)$. Moreover, the survival rate was significantly improved by the regular periodic follow-up $(P=0.03)$. The patients experiencing periodic follow-up under the supervision of oral hygienists had no significant difference in the survival rate $(P=0.51)$. The survival rate showed no significant relationship with the patient delay $>4$ weeks $(P=0.37)$, and also with the professional delay $(P=0.54)$, as well as with the degree of tumor differentiation $(P=0.35)$. According to Table 2 , the multivariate Cox regression revealed that the survival rate was not in significant relationship with the treatments of only surgery $(P=0.055)$, only radiotherapy $(P=0.64)$ and only chemotherapy $(P=0.73)$.

\section{Discussion}

This study examined the association between the demographic variables of oral SCC (OSCC) patients, tumor-related characteristics, and the used diagnostic and therapeutic methods with survival rate of patients. Data were collected from patients during 2009-2012 in Cancer Registry Center of Ministry of Health and Medical Education and Cancer Institute of Imam Khomeini Hospital, Iran. Based on the results, only age and periodic follow-ups showed a significant correlation with the survival rate. This study obtained 1-year survival rate of $91.46 \%$ with a standard error (SE) of 3.09, 2-year overall survival rate of $69.51 \%(\mathrm{SE}=5.08)$, 3-year overall survival rate of $53.7 \%(\mathrm{SE}=5.5)$, and 5-year overall survival rate of $40.24 \%$ $(\mathrm{SE}=5.5)$. In a study by Warnakulasuriya et al. ${ }^{8} 5$-year overall survival rate in most countries for oral cancer was reported to be $50-60 \%$. In a study by Silverman ${ }^{9}$ in the United States, the 5 -year overall survival rate was $60 \%$, which is $10 \%$ higher than this study. The difference in the survival rate in this research with other studies in developed countries could be due to better health infrastructures of these countries or better access to treatment centers for the patients. In a study in Netherlands in 2014, the survival rate was improved in spite of the increased incidence of OSCC..$^{10}$ An increase in the incidence of cancer can be attributed to changes in lifestyle, and improved survival 
rate due to the development of health facilities and the promotion of public awareness.

In this study, the mean age of all patients with oral cancer was 66.1 years. In addition, $32.9 \%$ of subjects under study were aged $\leq 60$ years old and $67.1 \%$ were aged $>60$ years. Also, the minimum and maximum age of these patients was 31 and 89 years, respectively. In the study by Warnakulasuriya et al. ${ }^{8}$ in England in 2010, the mean age of patients was 62 years. In a study in 2013, the frequency of patients over the age of 40 years was about eight times more than people under 40 years.

Salian et al. ${ }^{6}$ reported a maximum outbreak in the $5^{\text {th }}$ and $6^{\text {th }}$ decades. In another study, the age range of affected individuals was reported between 11 and 94 years. The incidence of SCC is very rare in children and syndromes associated with genetic disorders can be raised in case of any incidence. ${ }^{1}$ In a study by Chamani ${ }^{11}$ in Iran, the mean age of patients with oral cancer was 11 years less than this study, which was 54.5 years. Bhurgri ${ }^{13}$ in India showed that only $2 \%$ of patients were over 60 years of age. In countries with high prevalence of oral cancers, it can be expected that the age of disease onset is lower than in other countries. Moreover, the lower life expectancy in these countries is effective in this age ratio. In recent decades, the prevalence of SCC in younger people has been considered, ${ }^{5}$ which could be due to changes in lifestyle for example the onset of tobacco and alcohol use and sexual intercourse at an earlier age. In this study, only the age variable showed a significant relationship with the survival rate of patients. Aging led to a decrease in the level of body immunity and an increase in the comorbidities such as cardiovascular, respiratory and renal diseases in patients with SCC, causing the reduction in patient survival. ${ }^{14}$ However, this relationship was not significant in the study of Sargeran ${ }^{15}$ in Iran, Chandu et al. ${ }^{16}$ in Australia and Chen et al. ${ }^{17}$ in Taiwan, as well as three other studies in 2014. ${ }^{18-20}$

This study showed a higher prevalence of OSCC in men. According to the results, $64.63 \%$ of the patients were male and the rest were female. In an epidemiological study in Argentina, the prevalence of oral cancer in men between 1992 and 2000 was 1.24 times higher than in women. ${ }^{21}$

In the study by Sargeran et al. ${ }^{22}$ in Iran, $55 \%$ of patients were male and the rest were women. In another study in Australia, $56.8 \%$ of the patients were male. ${ }^{16}$ In several other studies, male involvement was 1.5, 1.9, and 1.4 times higher than women. ${ }^{1,7,8}$ Meanwhile, an increase in the incidence of oral cancer in women has been noted in recent years. ${ }^{5,23}$ Two studies confirmed the higher prevalence of OSCC in women. ${ }^{24,25}$ Further, a study reported a steady increase in the incidence of SCC in middle-aged women over the past two decades, despite a lower incidence of oral cancer in women. ${ }^{23}$ Changes in the gender ratio in patients, especially in recent years, can be strongly attributed to changes in lifestyle, smoking habits, non-inhaled tobacco usage, alcohol consumption, hormonal changes, and nutritional deficiencies such as iron, riboflavin, vitamins and minerals in women. ${ }^{5,23}$ Since cigarette and alcohol habits are less common in Iranian women, further attention should be driven toward other predisposing factors, such as nutritional deficiencies, viral agents such as HPV and chronic trauma, to prevent female involvement with SCC. The relationship between gender variable and survival rate in this study was not significant, consistent with the studies by Sargeran, ${ }^{15}$ Chandu et al. ${ }^{16}$ and Chen et al. ${ }^{17}$ and Hanemann et al., ${ }^{19}$ Fan et al. ${ }^{18}$ and Fang et al. ${ }^{19}$ The responses to the therapeutic and clinical course of the disease appear not to be affected by gender factors, resulting in a lack of influence of the gender factor on the survival rate.

In this study, 33 patients (40.25\%) survived and the rest died. Of the 49 deceased patients with SCC, the cause of death was the cancer complications in $43(93.47 \%)$ patients and other causes in three (6.53\%) patients. In the study of Chandu et al., ${ }^{16}$ out of 115 patients, 88 (76.5\%) survived, and 27 died of which 17 (14.8\%) due to complications of cancer and 10 (8.7\%) for other causes. In the study of Sargeran, ${ }^{15}$ the causes for death in 470 patients were the complications of cancer in 335 (71\%) and the other causes in 18 (4\%). Of these patients, 80 (17\%) survived, and eventually $37(8 \%)$ were undiagnosed. Based on the results, it seems that other factors such as patient delay due to lack of knowledge or lack of timely access to diagnostic and therapeutic centers as well as professional delay can be considered as key factors in the high mortality rate of oral cancer.

In this study, the first patient referral was to specialist physician in $41(56.18 \%)$ out of all patients with SCC, a general practitioner in $24(32.87 \%)$ and a dentist in the rest (10.95\%). In the study of Kerdpon and Sriplung ${ }^{26}$ in Thailand, the first patient referral was to a practitioner in 133 patients (82.6\%), to a dentist in 25 (15.5\%) and to a public health care provider in three $(1.9 \%)$, reflecting differences in care and referral systems in Thailand. According to the results, it can be argued that the role of dentists has not been explained as the first referral in the diagnosis of OSCC in different societies, especially in our country, and inadequate information has been provided to society in this regard.

The most widely used treatment method for the patients among our statistical community was to combine all three methods of surgery, radiotherapy and chemotherapy. Among patients with OSCC, $37.5 \%$ experienced all three treatments, $12.5 \%$ surgery alone, $5.1 \%$ radiotherapy alone, $6.6 \%$ chemotherapy alone, $10.3 \%$ chemotherapy plus radiotherapy, $8.1 \%$ surgery plus chemotherapy and $11 \%$ radiotherapy plus surgery. In the study of Sargeran, ${ }^{15} 17 \%$ experienced only surgery, $66 \%$ surgery plus radiotherapy, and $17 \%$ radiotherapy alone. In this study, the treatment method used had a significant relationship with survival.

In the study of Chen et al., ${ }^{17}$ the surgical treatment method had a significant relationship with survival rate, but not significant in this study though the $P$-value was very close to the significance level (0.055), indicating the importance of surgical therapy in the OSCC treatment. This method, combined with other methods, leads to improved patient survival.

Hasegawa et al. ${ }^{27}$ also showed significantly higher loco regional control in patients treated by combining three therapies compared with surgery alone and surgery with radiotherapy. However, there was no significant difference in the overall survival rate of patients who received radiotherapy compared with the group receiving all three therapies.

In this study, it has been shown that regular periodic follow-ups by physicians, in contrast to orodental health technicians, have a significant effect on increasing the survival of patients through the timely diagnosis, treatment or referral of recurrent lesions.

Rapid diagnosis of cancer and urgent referral of patients are important factors in improving the survival rate of patients. ${ }^{28}$ In this study, the knowledge of patients about the symptoms of oral cancer as well as the status of the treatment 
system for early referral and treatment were investigated as similar to a study by Dios et al. ${ }^{29}$ The mean patient delay was 16.3 weeks, approximately 4 months, for the patients in this study. The minimum and maximum patient delay was 1 week and 1 year ( 52 weeks) with a standard deviation of 17.7 weeks. In this study, 37.8 patients with OSCC had a delay of $<4$ weeks, and 46.3 patients had a delay of $>4$ weeks. In the study of Brouha et al. ${ }^{28}$ in The Netherlands, $47 \%$ of patients had a delay of $<4$ weeks. In the study of Kerdpon and Sriplung, ${ }^{26}$ the minimum and maximum patient delay was 0 day and 102 weeks. In this study, the mean patient delay was reported to be 13 weeks. The mean patient delay for this study was 3 weeks longer. A higher patient delay and higher percentages of patients with a delay of $>4$ weeks can highlight the low level of awareness of cancer patients or lack of access to specialized centers for the diagnosis and treatment of cancer. A quiet clinical course in oral cancer, which is lacking in symptoms such as pain or paresthesia and bleeding until late advanced stages, can be one of the important reasons for delaying the patient referral for diagnosis.

In this study, the mean professional delay was 4.75 weeks, with a minimum and maximum professional delay of 1 week and 28 weeks. The standard deviation for professional delay was 5.29 weeks. In the study of Kerdpon and Sriplung in Thailand, the minimum and maximum professional delay was 0 day and 104 weeks. In their study, the mean professional delay was reported to be 7 weeks. ${ }^{26}$ The highly diverse clinical presentations of OSCC, which can be mistaken for differential diagnosis with irritative and benign oral lesions, are among the important reasons raised in professional delay, suggesting the need for specialist retraining courses for the differentiation of clinical symptoms of oral cancer for physicians, general dentists, and specialized dentists.

In this study, the most common location of tumor was the lateral borders of tongue (with a prevalence of 20.4\%) and the least affected areas $(0.9 \%)$ were gums and palate, in line with the findings of the study of Juan-Carlos as the tongue (44.7\%) showed the most prevalent involved regions and the palate $(0.5 \%)$ had the minimum involvement. ${ }^{7}$ In the review study of Gupta et al., ${ }^{5}$ the tongue was the most commonly involved location in the European, American and Brazilian populations, while buccal mucosa and tongue were among the most commonly reported sites in Southeast Asia according to the patient habit of using chewing tobacco. In the study of Gehani and Goteti ${ }^{1}$ in Libya, the tongue was reported as the most commonly involved site. The tongue and the floor of mouth are known as a premalignant condition in the oral cavity as these areas had higher loss of heterozygosity than other areas of the mouth. Moreover, more contact and penetration of the carcinogens in foods and tobacco could be suggested as a cause for higher incidence of cancer in these areas.

In this study, most of SCCs had good degree of differentiation at diagnosis (67.39\%), and the degree of tumor differentiation showed no significant relationship with survival rates. In the study of Juan-Carlos, the highest rate was related to the moderate degree of differentiation (61.2\%). In their study, the survival rate was significantly correlated with degree of tumor differentiation, indicating a high percentage of patients with moderate degree of differentiation, poor quality of life and low survival rate. ${ }^{7}$ The degree of tumor differentiation depends on many factors at the time of diagnosis, including professional delay, patient delay, age, tumor location, and other cases. In the study of Chen et al., ${ }^{17}$ the association between histological differentiation and survival rate was significant, but not significant in this study.

\section{Conclusion}

Considering the increased percentage of people with oral cancer, it is essential to get knowledge about the epidemiologic conditions of these patients, to investigate the barriers and problems in the health care system of different societies to improve the quality-of-life of these patients, and to develop educational programs. Oral cancers require special attention due to high prevalence and overall survival rates. Training oral health care providers, especially dentists, on precise examination and early detection of lesions and periodic follow-up of patients after histopathological diagnosis can be an important step in increasing the survival of these patients. Moreover, registration of treatment methods and response rates to these methods can be the basis for oral health decision making.

\section{Conflicts of Interest}

None.

\section{References}

1. Gehani RE, Goteti SHL. Squamous cell carcinoma of oral cavity: changing trends. J Dent Health Oral Disord Ther. 2015;2:00051.

2. Perera M, Al-Hebshi NN, Perera I, Ipe D, Ulett GC, Speicher DJ, et al. Inflammatory bacteriome and oral squamous cell carcinoma. J Dent Res. 2018;97:725-732.

3. Ehtesham H, Safdari R, Mansourian A, Tahmasebian S, Mohammadzadeh N, Ghazisaeedi M, et al. Clinical decision support system, a potential solution for diagnostic accuracy improvement in oral squamous cell carcinoma: a systematic review. J Oral Health Oral Epidemiol. 2017;6:187-195.

4. Jafari A, Gholizadeh N, Ramezani R, Razavi HE, Najafi S. Evaluation of survival rate in patients with laryngeal cancer. J Dent Med. 2017;30:89-96.

5. Gupta N, Gupta R, Acharya AK, Patthi B, Goud V, Reddy S, et al. Changing trends in oral cancer - a global scenario. Nepal J Epidemiol. 2016;6:613-619.

6. Salian V, Dinakar C, Shetty P, Ajila V. Etiological trends in oral squamous cell carcinoma: a retrospective institutional study. Cancer Transl Med. 2016;2:33-36

7. Hernández-Guerrero JC, Jacinto-Alemán LF, Jiménez-Farfán MD, MacarioHernández A, Hernández-Flores F, Alcántara-Vázquez A. Prevalence trends of oral squamous cell carcinoma. Mexico City's General Hospital experience. Med Oral Patol Oral Cir Bucal. 2013;18:e306-e311.

8. Warnakulasuriya S. Living with oral cancer: epidemiology with particular reference to prevalence and life-style changes that influence survival. Oral Oncol. 2010:46:407-410

9. Silverman S Jr, Kerr AR, Epstein JB. Oral and pharyngeal cancer control and early detection. J Cancer Educ. 2010;25:279-281.

10. Braakhuis BJ, Leemans CR, Visser O. Incidence and survival trends of head and neck squamous cell carcinoma in the Netherlands between 1989 and 2011. Oral Oncol. 2014;50:670-675.

11. Maleki D, Ghojazadeh M, Mahmoudi SS, Mahmoudi SM, Pournaghi Azar F, Torab A, Piri R, Azami Aghdash S, Naghavi Behzad M. Epidemiology of oral cancer in Iran: a systematic review. Asian Pacific Journal of Cancer Prevention. 2015;16:5427-32.

12. Hwa JS, Kwon OJ, Park JJ, Woo SH, Kim JP, Ko GH, et al. The prognostic value of immunohistochemical markers for oral tongue squamous cell carcinoma. Eur Arch Otorhinolaryngol. 2015;272:2953-2959.

13. Bhurgri Y. Cancer of the oral cavity-trends in Karachi South (1995-2002). Asian Pac J Cancer Prev. 2005:6:22-26.

14. Golizadeh N, Najafi Sh, Khayamzadeh M, Afzali S, Sheykhbahaei N. Trend in laryngeal cancer, mortality and survival rate in Iran. J Contemp Med Sci. 2018:4:7-11. 
15. Sargeran K. Oral Cancer in Tehran, Iran: an approach for understanding disease burden. 2008

16. Chandu A, Adams G, Smith AC. Factors affecting survival in patients with oral cancer: an Australian perspective. Int J Oral Maxillofac Surg. 2005:34:514-520.

17. Chen YK, Huang HC, Lin LM, Lin CC. Primary oral squamous cell carcinoma: an analysis of 703 cases in southern Taiwan. Oral Oncol. 1999;35:173-179.

18. Fan Y, Zheng L, Mao MH, Huang MW, Liu SM, Zhang J, et al. Survival analysis of oral squamous cell carcinoma in a subgroup of young patients. Asian Pac J Cancer Prev. 2014;15:8887-8891.

19. Fang QG, Shi S, Liu FY, Sun CF. Squamous cell carcinoma of the oral cavity in ever smokers: a matched-pair analysis of survival. J Craniofac Surg. 2014;25:934-937.

20. Hanemann JA, Oliveira DT, Nonogaki S, Nishimoto IN, de Carli ML, Landman $\mathrm{G}$, et al. Expression of E-cadherin and B-catenin in basaloid and conventional squamous cell carcinoma of the oral cavity: are potential prognostic markers? BMC Cancer. 2014;14:395.

21. Brandizzi D, Chuchurru JA, Lanfranchi HE, Cabrini RL. Analysis of the epidemiological features of oral cancer in the city of Buenos Aires. Acta Odontol Latinoam. 2005:18:31-35.

22. Sargeran K, Murtomaa H, Safavi SMR, Vehkalahti MM, Teronen O. Survival after diagnosis of cancer of the oral cavity. Br J Oral Maxillofac Surg. 2008;46:187-191.
23. La Vecchia C, Lucchini F, Negri E, Levi F. Trends in oral cancer mortality in Europe. Oral Oncol. 2004:40:433-439.

24. Muscat JE, Richie JP Jr, Thompson S, Wynder EL. Gender differences in smoking and risk for oral cancer. Cancer Res. 1996;56:5192-5197.

25. Vatanasapt P, Suwanrungruang K, Kamsa-Ard S, Promthet S, Parkin MD. Epidemiology of oral and pharyngeal cancers in Khon Kaen, Thailand: a high incidence in females. Asian Pac J Cancer Prev. 2011;12:2505-2508.

26. Kerdpon D, Sriplung H. Factors related to delay in diagnosis of oral squamous cell carcinoma in southern Thailand. Oral Oncol. 2001;37:127-31.

27. Hasegawa T, Yanamoto S, Otsuru M, Yamada SI, Minamikawa T, Shigeta T, et al. Retrospective study of treatment outcomes after postoperative chemoradiotherapy in Japanese oral squamous cell carcinoma patients with risk factors of recurrence. Oral Surg Oral Med Oral Pathol Oral Radiol. 2017;123:524-530

28. Brouha XD, Tromp DM, Hordijk GJ, Winnubst JA, de Leeuw JR. Oral and pharyngeal cancer: analysis of patient delay at different tumor stages. Head Neck. 2005:27:939-945

29. Diz Dios P, Padrón González N, Seoane Lestón J, Tomás Carmona I, Limeres Posse J, Varela-Centelles P."Scheduling Delay" in oral cancer diagnosis: a new protagonist. Oral Oncol. 2005;41:142-146.

This work is licensed under a Creative Commons Attribution-NonCommercial 3.0 Unported License which allows users to read, copy, distribute and make derivative works for non-commercial purposes from the material, as long as the author of the original work is cited properly. 